\title{
Expedient Synthesis of 3-Benzoylflavones by PCC Oxidation of 3-Benzylideneflavanones
}

\author{
Se Hee Kim, Sung Hwan Kim, and Jae Nyoung Kim* \\ Department of Chemistry and Institute of Basic Science, Chonnam National University, Guangiu 500-757, Korea \\ E-mall:kimmaichonnamac.kr \\ Received July: 29, 2008
}

Key Words : Baylis-Hillnan adducts, Flavanones, PCC., Flavones

The synthesis and chemical transformation of 3-arylideneflavanones (3-arylidenechroman-4-ones) and related compounds received much attention due to the abundance of this moiety in many natural products and biologically active substances. ${ }^{1-3}$ Many 3-arylideneflavanones showed interesting biological activities including anti-HIV, anti-mutagenic, anti-inflammatory, anti-bacterial, anti-fungal and antiviral activities. ${ }^{1-3}$ In addition, oxidation of 3-arylideneflavanones into 3-aroylflavones (3-aroylchromones) ${ }^{3}$ is also regarded as an important transtormation in this respect.

In this paper, we described the synthesis of various 3 benzylideneflavanones 6 and the following oxidation with pyridinium chlorochromate ( $\mathrm{PCC}$ ) to make the corresponding 3-benzoylflavones 7 (Scheme 1 and Table 1). The synthesis of 3-arylidenetlavanones 6 was carried out by tollowing the method of Basavaiah ${ }^{16}$ from the Baylis-Hillman adducts $^{4,5}$ via the following three-step sequence comprised of (i) introduction of phenol at the primary position of the Baylis-Hillman adduct, (ii) hydrolysis of the ester group and (iii) Friedel-Crafts type cyclization. ${ }^{\text {lb-d }}$

The starting material 4a was synthesized in pure $E$-form in good yield $(94 \%)$ by the reaction of phenol (3a) and the cinnamyl bromide $2{ }^{1 \mathrm{~b}-\mathrm{d}, \mathrm{t}}$ which was easily prepared from 1 and $\mathrm{HBr}$, under the influence of $\mathrm{K}_{2} \mathrm{CO}_{3}$ in acetone. Hydrolysis of $4 \mathrm{a}$ was carried out in aqueous $\mathrm{KOH}$ to produce the corresponding acid 5a. Without further purification, treatment of $\mathbf{5} \mathbf{a}$ with trifluoroacetic anhydride (TFAA) produced 3 -benzylideneflavanone $6 \mathrm{a}$ in $90 \%$ yield. ${ }^{15}$ With this compound in our hand we examined the oxidation of $6 \mathrm{a}$ with PCC which was found as an effective oxidant in a similar system by us recently. ${ }^{6}$ As expected, treatment of 6 a with
PCC (5.0 equiv) in DMF afforded 3-benzoyltlavone $7 \mathrm{a}^{3.7}$ in moderate yield $(56 \%)$, although long reaction time $(72 \mathrm{~h})$ was required for the oxidation.

Encouraged by the results we prepared starting materials 4b-g (80-91\%) from the reactions of 2 and 4-methylphenol (3b), 2-methylphenol (3c), 4-methoxyphenol (3d), 3,5dimethylphenol (3e), 1-naphthol (3f), and 2-naphthol (3g). By following the same procedure of $6 \mathbf{a}$ we synthesized various 3-benzylidenetlavanones $6 \mathbf{b}-\mathbf{g}$ as summarized in Table 1. As shown in entry 7 , the cyclization reaction of compound $\mathbf{4 g}$ occurred at the 1-position of naphthalene moiety selectively and produced $6 \mathrm{~g}$. PCC oxidation of $6 \mathrm{~b}-\mathrm{g}$ was also carried out and desired 3-benzoyltlavones $7 \mathrm{~b}-\mathrm{g}$ were prepared in $46-70 \%$ yields. Similarly, we synthesized nitrogen analog 8 with $N$-tosylaniline as in Scheme 2. Compound 9 was synthesized by using the same protocol of 6a-g, however, the oxidation of 9 was failed. Double bond isomerization of 6 a from the exo- to the endo-position was also examined (Scheme 3). Initially, we tried the isomerization under catalytic hydrogenation conditions ${ }^{8-c}$ and obtained desired compound 3-benzylflavone $(10)^{8 b}$ in low yield $(37 \%)$ due to the formation of fully reduction compound $11(32 \%))^{8 b}$ In addition, the ratio of $10 / 11$ was highly dependent on the reaction conditions and it was difficult to make $\mathbf{1 0}$ as the major product. After many trials, we found that 10 can be prepared from 6a in good yield $(71 \%)$ under the influence of DBU ( 1.2 equiv) in $\mathrm{CH}_{3} \mathrm{CN}\left(40^{\circ} \mathrm{C}, 12 \mathrm{~h}\right)$.

In summary, we disclosed a facile synthesis of 3-benzylideneflavanones and 3-benzoylflavones from Baylis-Hillman adducts. The biological activities of synthesized compounds will be examined and published in due course.

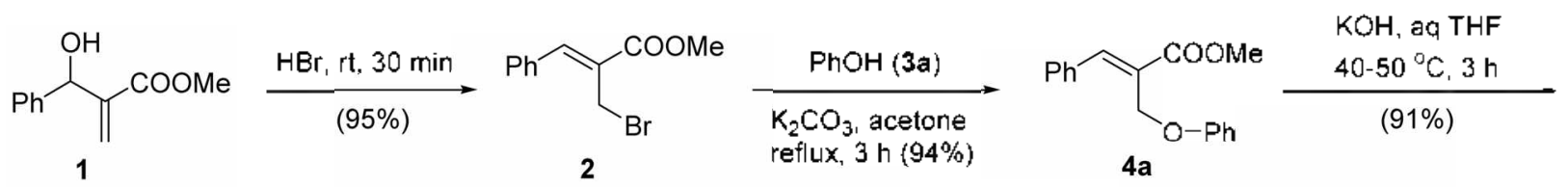

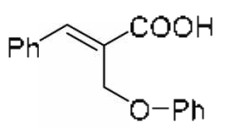

5 a

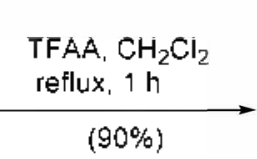

$(90 \%)$

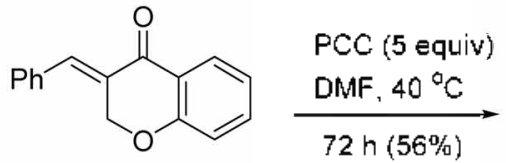

$6 a$

3-benzylideneflavanone

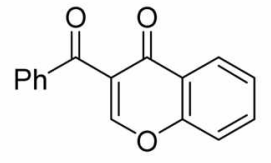

$7 a$

3-benzoylflavone

Scheme 1 
Table 1. Synthesis of 3-benzylideneflavanones and 3-benzoylflavones

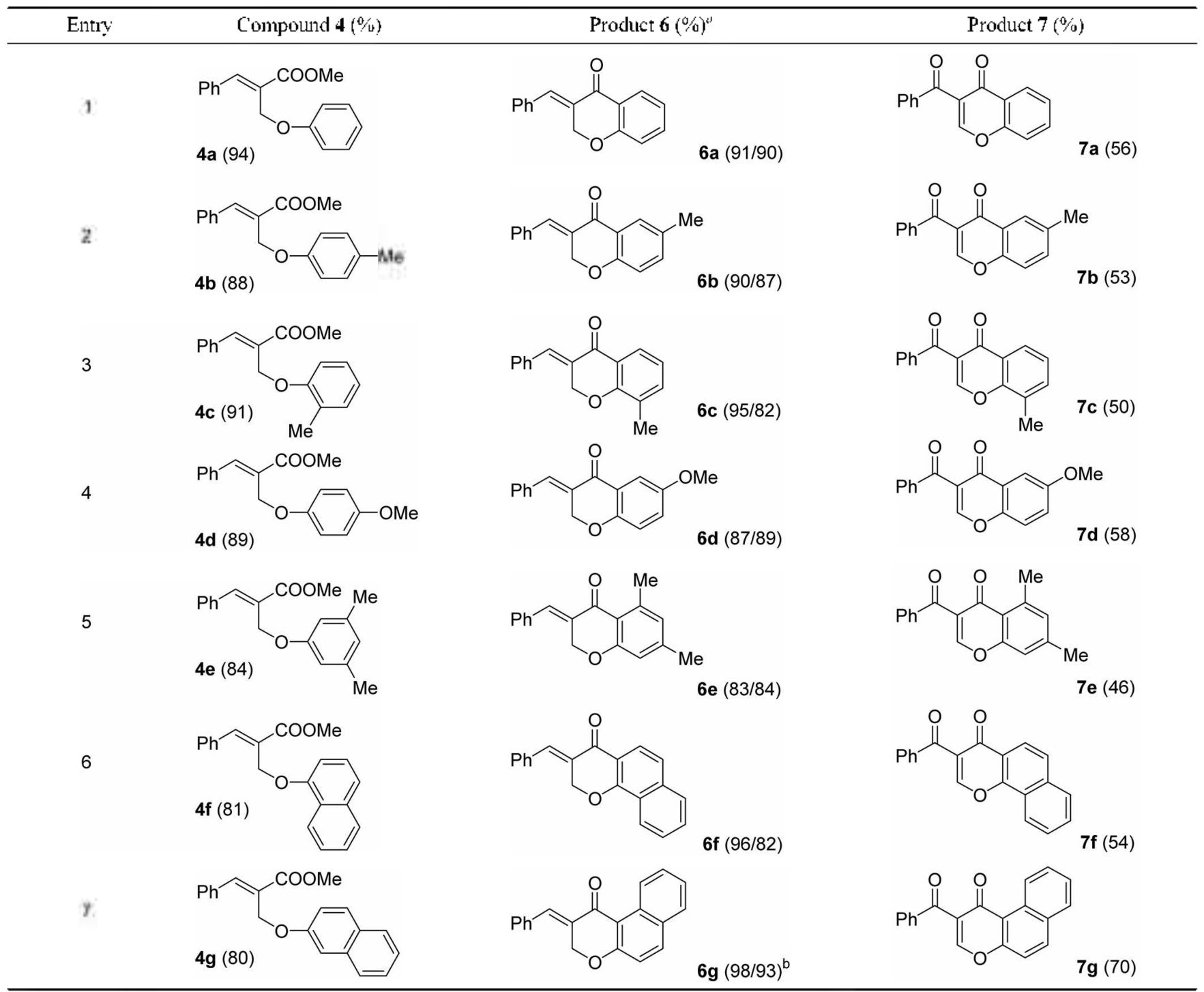

"First yields refer to hydrolysis stage to compounds $\mathbf{5 a - g}$ and the second yields to cyclization step to $\mathbf{6 a - g} .{ }^{\text {th }}$ The stucture was confirmed by the splitting pattern of aromatic protons in 'H NMR (Experimental)

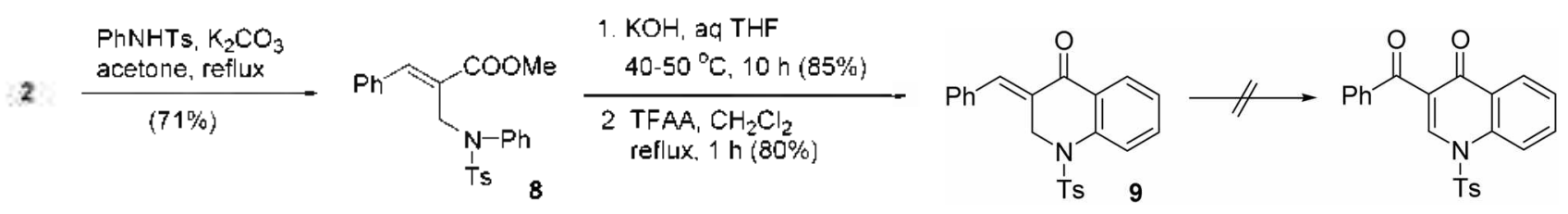

Scheme 2<smiles>O=c1c(Cc2ccccc2)coc2ccccc12</smiles>

$10(71 \%)$

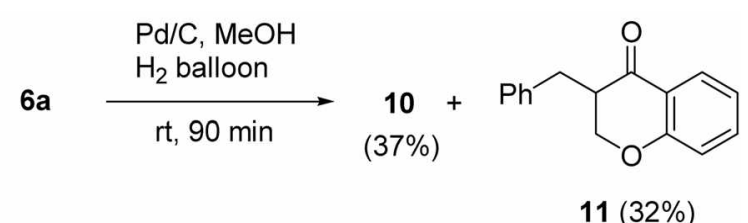

Scheme 3

\section{Experimental Section}

Typical procedure for the synthesis of $4 a$. Baylis-Hillman adduct 1 (384 mg. $2.0 \mathrm{mmol}$ ) was treated with aqueous $\mathrm{HBr}$
$(48 \%, 2.0 \mathrm{~mL})$ at room temperature for $30 \mathrm{~min}$. After the usual extractive workup with ether and column chromatographic purification (hexanes/EtOAc, 8:1) process, cinnamyl bromide 2 was obtained as colorless oil, $485 \mathrm{mg}(95 \%)$. 
The reaction mixture of 2 (255 $\mathrm{mg}, 1.0 \mathrm{mmol}$ ), phenol (3a. $104 \mathrm{mg}, 1.1 \mathrm{mmol}$, and $\mathrm{K}_{2} \mathrm{CO}_{3}(207 \mathrm{mg}, 1.5 \mathrm{mmol})$ in acetone $(5 \mathrm{~mL})$ was heated to reflux for $3 \mathrm{~h}$. After the usual extractive workup with ether and column chromatographic purification (hexanes/EtOAc, 5:1) process, compound $\mathbf{4 a}$ was obtained as colorless oil, $252 \mathrm{mg}(94 \%)$. Other products including 8 were prepared analogously and the spectroscopic data of $\mathbf{4 c}, \mathbf{4 d}, \mathbf{4 f}, \mathbf{4 g}$, and 8 are as follows. Com-

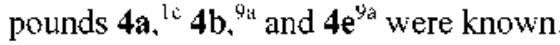

Compound 4c: $91 \%$; colorless oil; IR (film) 1718, 1495. $1234,1117 \mathrm{~cm}^{-1}$; ${ }^{1} \mathrm{H}$ NMR $\left(\mathrm{CDCl}_{3}, 300 \mathrm{MHz}\right) \delta 2.22(\mathrm{~s}$, $3 \mathrm{H}), 3.84$ (s, $3 \mathrm{H}), 4.84(\mathrm{~s}, 2 \mathrm{H}), 6.87-6.91(\mathrm{~m}, 2 \mathrm{H}), 7.13-7.17$ $(\mathrm{m}, 2 \mathrm{H}), 7.35-7.37(\mathrm{~m}, 3 \mathrm{H}), 7.49-7.51(\mathrm{~m}, 2 \mathrm{H}), 8.04(\mathrm{~s}, 1 \mathrm{H})$; ${ }^{13} \mathrm{C}$ NMR $(\mathrm{CDCl}, 35 \mathrm{MHz}) \delta 16.27,52.22,62.89,111.80$. $120.79,126.74,127.33,127.59,128.66,129.51,129.69$. $130.68,134.52,145.32,156.68,167.77$.

Compound 4d: $89 \%$; colorless oil; IR (film) 1718, 1508 . $1225 \mathrm{~cm}^{-1}$; ${ }^{1} \mathrm{H} \mathrm{NMR}\left(\mathrm{CDCl}_{3}, 500 \mathrm{MHz}\right) \delta 3.77$ (s. 3H), 3.84 (s, $3 \mathrm{H}), 4.78(\mathrm{~s}, 2 \mathrm{H}), 6.85(\mathrm{~d}, J=9.0 \mathrm{~Hz}, 2 \mathrm{H}), 6.92(\mathrm{~d}, J=$ $9.0 \mathrm{~Hz}, 2 \mathrm{H}), 7.35-7.38(\mathrm{~m}, 3 \mathrm{H}), 7.48-7.50(\mathrm{~m}, 2 \mathrm{H}), 8.03(\mathrm{~s}$. $1 \mathrm{H}) ;{ }^{13} \mathrm{C} \mathrm{NMR}\left(\mathrm{CDCl}_{3}, 125 \mathrm{MHz}\right) \delta 52.24,55.66,63.52$. $114.60,116.09,127.44,128.65,129.53,129.74,134.43$. $145.45,152.58,154.15,167.67$

Compound 4f: 81\%; colorless oil; [R (film) 1716, 1267. $1235,1094 \mathrm{~cm}^{-1} ;{ }^{1} \mathrm{H}$ NMR $\left(\mathrm{CDCl}_{3}, 300 \mathrm{MHz}\right) \delta 3.85$ (s, $3 \mathrm{H}), 5.01$ (s, $2 \mathrm{H}), 6.87$ (d, $J=7.5 \mathrm{~Hz}, 1 \mathrm{H}), 7.31-7.39(\mathrm{~m}$. $4 \mathrm{H}), 7.43-7.55(\mathrm{~m}, 5 \mathrm{H}), 7.81$ (d, $J=7.5 \mathrm{~Hz}, 1 \mathrm{H}), 8.13$ (s, $1 \mathrm{H}), 8.27(\mathrm{~d}, J=8.5 \mathrm{~Hz}, 1 \mathrm{H}) ;{ }^{13} \mathrm{C} \times \mathrm{MR}(\mathrm{CDCl}, 75 \mathrm{MHz}) \delta$ $52.34,63.00,105.24,120.64,122.25,125.23,125.83$. $126.42,127.33,127.38,128.55,128.75,129.60,129.73$, $134.48,134.52,145.82,154.25,167.77$.

Compound 4g: 80\%; colorless oil; IR (film) 1717, 1629. $1256,1234,1214 \mathrm{~cm}^{-1} ;{ }^{1} \mathrm{H}$ 入MR $\left(\mathrm{CDCl}_{3}, 300 \mathrm{MHz}\right) \delta 3.86$ (s, $3 \mathrm{H}), 4.96(\mathrm{~s}, 2 \mathrm{H}), 7.18-7.25(\mathrm{~m}, 2 \mathrm{H}), 7.32-7.37(\mathrm{~m}, 4 \mathrm{H})$, $7.41-7.51(\mathrm{~m}, 3 \mathrm{H}), 7.70(\mathrm{~d}, J=8.1 \mathrm{~Hz}, 1 \mathrm{H}), 7.77(\mathrm{~d}, J=9.0$ $\mathrm{Hz}, 1 \mathrm{H}), 7.78(\mathrm{~d}, J=8.1 \mathrm{~Hz}, 1 \mathrm{H}), 8.09(\mathrm{~s}, 1 \mathrm{H}) ;{ }^{13} \mathrm{C}$ NMR $\left(\mathrm{CDCl}_{\text {: }}, 75 \mathrm{MHz}\right) \delta 52.35,62.78,107.23,119.15,123.75$. $126.38,126.79,127.17,127.63,128.75,129.15,129.43$, $129.64,129.76,134.42,134.47,145.83,156.36,167.67$.

Compound 8: 71\%; colorless oil; $\mathbb{R}$ (film) 1716, 1352, $1253,1165 \mathrm{~cm}^{-1} ;{ }^{1} \mathrm{H}$ XVR (CDCl $\left.3,300 \mathrm{MHz}\right) \delta 2.38(\mathrm{~s}$, $3 \mathrm{H}), 3.65(\mathrm{~s}, 3 \mathrm{H}), 4.71(\mathrm{~s}, 2 \mathrm{H}), 6.73(\mathrm{~d}, J=7.5 \mathrm{~Hz}, 2 \mathrm{H})$, $7.08-7.40(\mathrm{~m}, 12 \mathrm{H}), 7.66(\mathrm{~s}, 1 \mathrm{H}) ;{ }^{13} \mathrm{C} \mathrm{NMR}\left(\mathrm{CDCl}_{3}, 75\right.$ $\mathrm{MHz}) \delta 21.54,46.16 .52 .09,126.81,127.90,128.05,128.44$ (2C), 129.17 (2C) $129.29,129.81,134.25,134.48,138.58$, $143.48,143.80,167.83$.

Typical procedure for the synthesis of $6 \mathrm{a}$. A mixture of 4a (268 $\mathrm{mg}, 1.0 \mathrm{mmol}$ ) and $\mathrm{KOH}(190 \mathrm{mg}, 3.0 \mathrm{mmol})$ in aqueous THF ( $3 \mathrm{~mL}$ ) was heated to $40-50^{\circ} \mathrm{C}$ for $3 \mathrm{~h}$. After acidification with aqueous $\mathrm{HCl}$ solution and the usual extractive workup with EtOAc, crude acid $\mathbf{5 a}$ was obtained in $91 \%$ yield $(232 \mathrm{mg}$ ). The acid $\mathbf{5 a}$ was used without further purification. A stirred solution of 5 a $(232 \mathrm{mg}, 0.91 \mathrm{mmol})$ and TFAA (390 $\mathrm{mg}, 1.86 \mathrm{mmol})$ in $\mathrm{CH}_{2} \mathrm{Cl}_{2}(5 \mathrm{~mL})$ was heated to reflux for $1 \mathrm{~h}$. After the usual extractive workup with ether and column chromatographic purification (hexanes/EtOAc, 4:1) process, compound 6a was obtained as yellow oil, $193 \mathrm{mg}(90 \%)$. Other compounds including 9 were prepared analogously and the spectroscopic data of $6 \mathbf{c}$, $6 \mathrm{e}-\mathrm{g}$, and 9 are as follows. Compounds $6 \mathrm{a},{ }^{\mathrm{k}} 6 \mathrm{~b},{ }^{9 \mathrm{~b}}$ and $6 \mathrm{~d}^{9 \mathrm{c}}$ were known.

Compound 6c: 82\%; yellow oil; IR (film) 1672, 1601 , 1479, $1304 \mathrm{~cm}^{-1} ;{ }^{1} \mathrm{H}$ NMR $\left(\mathrm{CDCl}_{3}, 300 \mathrm{MHz}\right) \delta 2.22(\mathrm{~s}$, $3 \mathrm{H}), 5.37(\mathrm{~d}, J=2.0 \mathrm{~Hz}, 2 \mathrm{H}), 6.96(\mathrm{t}, J=7.5 \mathrm{~Hz}, 1 \mathrm{H}), 7.31$ $7.35(\mathrm{~m}, 3 \mathrm{H}), 7.38-7.46(\mathrm{~m}, 3 \mathrm{H}), 7.87(\mathrm{~s}, 1 \mathrm{H}), 7.88(\mathrm{~d}, J=$ $9.0 \mathrm{~Hz}, 1 \mathrm{H}) ;{ }^{13} \mathrm{C} \times \mathrm{MR}\left(\mathrm{CDCl}_{3}, 75 \mathrm{MHz}\right) \delta 15.46,67.43$, $121.26,121.61,125.49,127.08,128.64,129.34,129.92$, $131.02,134.42,136.67,137.13,159.30,182.62$.

Compound 6e: $84 \%$; yellow solid, mp $74-76{ }^{\circ} \mathrm{C}$; IR (KBr) $1668,1614,1321,1165 \mathrm{~cm}^{-1}$; ${ }^{l} \mathrm{H}$ NMR $\left(\mathrm{CDCl}_{3}, 500\right.$ $\mathrm{MHz}) \delta 2.30(\mathrm{~s}, 3 \mathrm{H}), 2.69(\mathrm{~s}, 3 \mathrm{H}), 5.23(\mathrm{~d}, J=1.5 \mathrm{~Hz}, 2 \mathrm{H})$, $6.65(\mathrm{~s}, 1 \mathrm{H}), 6.69(\mathrm{~s}, 1 \mathrm{H}), 7.29(\mathrm{~d}, J=7.5 \mathrm{~Hz}, 2 \mathrm{H}), 7.37-7.45$ $(\mathrm{m}, 3 \mathrm{H}), 7.82(\mathrm{~s}, 1 \mathrm{H}) ;{ }^{13} \mathrm{C} \mathrm{NMR}\left(\mathrm{CDCl}_{3}, 125 \mathrm{MHz}\right) \delta 21.65$, $22.70,67.20,116.05,118.69,126.61,128.61,129.12$, $129.80,132.39,134.70,136.25,142.59,145.83,162.40$, 182.99; ESIMS $m / z 265.46\left(\mathrm{M}^{+}+1\right)$.

Compound 6f: $82 \%$; yellow solid, mp 78-80 ${ }^{\circ} \mathrm{C}$; IR (KBr) $1665,1625,1101 \mathrm{~cm}^{-1} ;{ }^{1} \mathrm{H} \times \mathrm{XM}\left(\mathrm{CDCl}_{3}, 300 \mathrm{MHz}\right) \delta 5.59$ $(\mathrm{d}, J=1.8 \mathrm{~Hz}, 2 \mathrm{H}), 7.34-7.65(\mathrm{~m}, 8 \mathrm{H}), 7.80(\mathrm{~d}, J=8.4 \mathrm{~Hz}$, $1 \mathrm{H}), 7.93(\mathrm{t}, J=1.8 \mathrm{~Hz}, 1 \mathrm{H}), 8.01(\mathrm{~d}, J=9.0 \mathrm{~Hz}, 1 \mathrm{H}), 8.26$ $(\mathrm{d}, J=8.4 \mathrm{~Hz}, 1 \mathrm{H}) ;{ }^{13} \mathrm{C} \mathrm{XMR}\left(\mathrm{CDCl}_{3}, 75 \mathrm{MHz}\right) \delta 68.29$, $116.35,121.53,122.54,123.43,124.82,126.22,127.88$, $128.72,129.36,129.65,129.94,130.49,134.49,137.01$, $137.40,159.26,181.83$

Compound 6g: 93\%; yellow solid, mp 66-68 ${ }^{\circ} \mathrm{C}$; IR (KBr) 1663, 1617, 1597, 1511, 1434, $1241 \mathrm{~cm}^{-1}$; ${ }^{1} \mathrm{H} \times \mathrm{MR}$ $\left(\mathrm{CDCl}_{3}, 300 \mathrm{MHz}\right) \delta 5.39(\mathrm{~d}, J=1.8 \mathrm{~Hz}, 2 \mathrm{H}), 7.09$ (d, $J=$ $9.3 \mathrm{~Hz}, 1 \mathrm{H}), 7.32-7.49(\mathrm{~m}, 6 \mathrm{H}), 7.67(\mathrm{t}, J=8.1 \mathrm{~Hz}, 1 \mathrm{H}), 7.77$ (d, $J=8.1 \mathrm{~Hz}, 1 \mathrm{H}), 7.92(\mathrm{~d}, J=8.4 \mathrm{~Hz}, 1 \mathrm{H}), 7.94(\mathrm{~s}, 1 \mathrm{H})$, $9.45(\mathrm{~d}, J=8.4 \mathrm{~Hz}, 1 \mathrm{H}) ;{ }^{13} \mathrm{C} \mathrm{NMR}\left(\mathrm{CDCl}_{3}, 75 \mathrm{MHz}\right) \delta$ $67.46,114.30,118.72,125.02,126.46,128.47,128.69$, $129.23,129.51,129.61,129.83,131.92,132.17,134.70$, 136.76, 137.41, 163.18, 182.54; ESMS m/z $287.44\left(\mathrm{M}^{-}+1\right)$.

Compound $9:^{9 \mathrm{~d}} 80 \%$; yellow solid, mp 135-137 ${ }^{\circ} \mathrm{C}$; IR (KBr) 1674, 1607, 1356, $1167 \mathrm{~cm}^{-1}$; ${ }^{1} \mathrm{H}$ NMR (CDCl, 300 $\mathrm{MHz}) \delta 2.35(\mathrm{~s}, 3 \mathrm{H}), 5.06(\mathrm{~d}, J=1.8 \mathrm{~Hz}, 2 \mathrm{H}), 6.97-7.04(\mathrm{~m}$, $4 \mathrm{H}), 7.32-7.54(\mathrm{~m}, 7 \mathrm{H}), 7.61-7.68(\mathrm{~m}, 1 \mathrm{H}), 7.83(\mathrm{~d}, J=8.7$ $\mathrm{Hz}, 1 \mathrm{H}), 7.95(\mathrm{dd}, J=7.8$ and $1.8 \mathrm{~Hz}, 1 \mathrm{H}) ;{ }^{13} \mathrm{C} \times \mathrm{MR}$ $\left(\mathrm{CDCl}_{3}, 75 \mathrm{MHz}\right) \delta 21.58,47.93,127.13,127.34,127.40$, $128.19,128.85,128.95,129.49,129.58,129.88,130.01$, $134.14,134.19,134.42,138.39,141.34,144.19,182.57$; ESIMS $m / z 390.49\left(\mathrm{M}^{+}+1\right)$.

Typical procedure for the synthesis of $7 \mathrm{a}$. A mixture of 6a (118 $\mathrm{mg}, 0.5 \mathrm{mmol}$ ) and $\mathrm{PCC}(540 \mathrm{mg}, 2.5 \mathrm{mmol})$ in DMF ( $2 \mathrm{~mL}$ ) was heated to $40^{\circ} \mathrm{C}$ for $72 \mathrm{~h}$. The reaction mixture was diluted with $\mathrm{CH}_{2} \mathrm{Cl}_{2}$ and filtered through a pad of Celite. After the usual aqueous extractive workup with $\mathrm{CH}_{2} \mathrm{Cl}_{2}$ and column chromatographic purification (hexanes/ EtOAc, 4:1) process, compound 7 a was obtained as a white solid, $70 \mathrm{mg}(56 \%)$. Other compounds were prepared analogously and the spectroscopic data of synthesized compounds $7 \mathbf{a}-\mathbf{g}$ are as follows.

Compound $7 \mathrm{a}^{7 \mathrm{~b}} 56 \%$; white solid, mp $128-130{ }^{\circ} \mathrm{C} ;{ }^{\mathrm{l}} \mathrm{H}$ NMR $\left(\mathrm{CDCl}_{3}, 300 \mathrm{MHz}\right) \delta 7.44-7.63(\mathrm{~m}, 5 \mathrm{H}), 7.72-7.78(\mathrm{~m}$, 
$1 \mathrm{H}), 7.85-7.88(\mathrm{~m}, 2 \mathrm{H}), 8.27$ (dd, $J=9.0$ and $1.2 \mathrm{~Hz}, 1 \mathrm{H}$ ), $8.30(\mathrm{~s}, 1 \mathrm{H}) ;{ }^{13} \mathrm{C} \times \mathrm{MR}(\mathrm{CDCl}, 75 \mathrm{MHz}) \delta 118.31,124.99$. $125.18,126.12,126.49,128.41,129.58,133.51,134.38$. $137.15,156.07,158.63 .174 .70,191.89$.

Compound $7 \mathrm{~b}$ : $53 \%$; white solid, mp $129-130^{\circ} \mathrm{C}$; IR (KBr) $1651,1618,1481,1319 \mathrm{~cm}^{-1}$; ${ }^{1} \mathrm{H}$ XMR (CDCl, 300 MHz) $\delta 2.47(\mathrm{~s}, 3 \mathrm{H}), 7.42-7.48(\mathrm{~m}, 3 \mathrm{H}), 7.52-7.61(\mathrm{~m}, 2 \mathrm{H})$, 7.84-7.88 (m, 2H), $8.03(\mathrm{~m}, 1 \mathrm{H}), 8.27(\mathrm{~s}, 1 \mathrm{H}) ;{ }^{13} \mathrm{C}$ NMR $\left(\mathrm{CDCl}_{;}, 75 \mathrm{MHz}\right) \delta 20.94,118.01,124.59,124.92,125.72$, $128.32,129.55,133.39,135.52,136.22,137.16,154.29$. $158.51,174.74,192.01$; ESIMS $m / z 265.40\left(\mathrm{M}^{-}+1\right)$.

Compound 7e: $50 \%$; white solid, mp $98-100{ }^{\circ} \mathrm{C}$; $\mathbb{R}$ (KBr) 1651, 1577, 1340, $1319 \mathrm{~cm}^{-1}$; ${ }^{1} \mathrm{H} \times \mathrm{MR}$ (CDCl; 300 MHz) $\delta 2.53$ (s, $3 \mathrm{H}), 7.36(\mathrm{t}, J=7.5 \mathrm{~Hz}, 1 \mathrm{H}), 7.43-7.49(\mathrm{~m}$. $2 \mathrm{H}), 7.56-7.62(\mathrm{~m}, 2 \mathrm{H}), 7.85-7.88(\mathrm{~m}, 2 \mathrm{H}), 8.08-8.11(\mathrm{~m}$. $1 \mathrm{H}), 8.34(\mathrm{~s}, 1 \mathrm{H}) ;{ }^{13} \mathrm{C} \mathrm{NMR}\left(\mathrm{CDCl}_{3}, 75 \mathrm{MHz}\right) \delta 15.48$. $124.01,124.90,124.92,125.60,127.85,128.37,129.55$. $133.43,135.33,137.20,154.58,158.36,175.03,192.03$.

Compound 7d: $58 \%$; white solid, $\mathrm{mp} 137-138{ }^{\circ} \mathrm{C}$; $\mathbb{R}$ (KBr) $1718,1508,1225 \mathrm{~cm}^{-1} ;{ }^{1} \mathrm{H} \times \mathrm{MR}\left(\mathrm{CDCl}_{3}, 300 \mathrm{MHz}\right)$ $\delta 3.90(\mathrm{~s}, 3 \mathrm{H}), 7.32(\mathrm{dd}, J=9.5$ and $3.5 \mathrm{~Hz}, 1 \mathrm{H}), 7.45-7.49$ $(\mathrm{m}, 3 \mathrm{H}), 7.58-7.62(\mathrm{~m}, 2 \mathrm{H}), 7.85-7.87(\mathrm{~m}, 2 \mathrm{H}), 8.29(\mathrm{~s}, 1 \mathrm{H})$; ${ }^{13} \mathrm{C} \mathrm{NMR}\left(\mathrm{CDCl}_{3}, 75 \mathrm{MHz}\right) \delta 55.96,105.57,119.70,124.31$ (2C) $125.72,128.37,129.55,133.41,137.27,150.86$. $157.61,158.42,174.56,192.12$

Compound 7e: $46 \%$; white solid, mp $153-155{ }^{\circ} \mathrm{C}$; $\mathbb{R}$ (KBr) $1658,1637,1596 \mathrm{~cm}^{-1}$; ${ }^{1} \mathrm{H} \times \mathrm{MR}$ (CDCl $3.300 \mathrm{MHz}$ ) $\delta 2.44(\mathrm{~s}, 3 \mathrm{H}), 2.78(\mathrm{~s}, 3 \mathrm{H}), 7.01$ (s. $1 \mathrm{H}), 7.15(\mathrm{~s}, 1 \mathrm{H}), 7.44-$ $7.47(\mathrm{~m}, 2 \mathrm{H}), 7.56-7.60(\mathrm{~m}, 1 \mathrm{H}), 7.85-7.87(\mathrm{~m}, 2 \mathrm{H}), 8.11(\mathrm{~s}$. $1 \mathrm{H}) ;{ }^{13} \mathrm{C}$ NMR $\left(\mathrm{CDCl}_{3}, 75 \mathrm{MHz}\right) \delta 21.57,22.78,116.10$. $120.98,126.09,128.40,129.48,130.06,133.35,137.38$. $141.53,144.57,156.60 .157 .65,176.65,192.42$.

Compound 7f: $54 \%$; white solid. mp $182-184{ }^{\circ} \mathrm{C}$ (decomp.); IR (KBr) 1662, 1641, $1392 \mathrm{~cm}^{-1}$; ${ }^{1} \mathrm{H}$ NMR (CDCl 3 . $300 \mathrm{MHz}) \delta 7.45-7.51(\mathrm{~m}, 2 \mathrm{H}), 7.59-7.64(\mathrm{~m}, 1 \mathrm{H}), 7.70-$ $7.79(\mathrm{~m}, 2 \mathrm{H}), 7.84(\mathrm{~d}, J=8.7 \mathrm{~Hz}, 1 \mathrm{H}), 7.90-7.93(\mathrm{~m}, 2 \mathrm{H})$. $7.96-7.99(\mathrm{~m}, 1 \mathrm{H}), 8.19(\mathrm{~d}, J=8.7 \mathrm{~Hz}, 1 \mathrm{H}), 8.47$ (s. $1 \mathrm{H})$, $8.53(\mathrm{~d}, J=7.8 \mathrm{~Hz}, 1 \mathrm{H}) ;{ }^{13} \mathrm{C}$ NMR $\left(\mathrm{CDCl}_{3}, 75 \mathrm{MHz}\right) \delta$ $120.89,121.52,122.23,123.77,126.22,126.44,127.58$, $128.21,128.46,129.64,129.77,133.59,136.11,137.08$, $153.64,157.46,174.56,191.92$.

Compound 7g: $70 \%$; white solid, mp $165-167{ }^{\circ} \mathrm{C}$; $\mathbb{R}$ (KBr) 1667, 1637, 1592, $1299 \mathrm{~cm}^{-1}$; ${ }^{1} \mathrm{H} \times \mathrm{MR}$ (CDCl, 300 MHz) $\delta 7.45-7.51(\mathrm{~m}, 2 \mathrm{H}), 7.54-7.67(\mathrm{~m}, 3 \mathrm{H}), 7.70-7.76(\mathrm{~m}$. $1 \mathrm{H}), 7.91-7.95(\mathrm{~m}, 3 \mathrm{H}), 8.15(\mathrm{~d}, J=9.0 \mathrm{~Hz}, 1 \mathrm{H}), 8.28$ (s, $1 \mathrm{H}), 9.94(\mathrm{~d}, J=8.4 \mathrm{~Hz}, 1 \mathrm{H}) ;{ }^{13} \mathrm{C} \times \mathrm{MR}\left(\mathrm{CDCl}_{3}, 75 \mathrm{MHz}\right) \delta$ $117.32,118.47,127.12,127.22,127.86,128.32,128.50$. $129.53,129.62,130.48,130.89,133.56,136.28,137.18$, 155.20, 157.47, 176.67, 192.31; ESIMS $m / z 301.42\left(\mathrm{M}^{-}+1\right)$.

Acknowledgments. This study was financially supported by Chonnam National University, 2007. Spectroscopic data was obtained from the Korea Basic Science Institute, Gwangju branch.

\section{References and Notes}

1. For the synthesis and biological activities of 3-arylideneflavanone derivatives, see: (a) Foroumadi, A.; Samzadeh-Kermani, A.; Emani, S.; Dehghan, G.; Sorkhi, M.; Arabsorkhi, F.; Heidari, M. R.; Abdollahi, M.; Shafiee, A. Bioorg. Med. Chent. Lett. 2007, 17, 6764-6769. (b) Rajan, Y. C.; Kanakam, C. C.; Selvam, S. P.; Murugesan, K. Tetrahedron Lett. 2007, 48, 8562-8565 and further references cited therein. (c) Basavaiah, D.; Bakthadoss, M.; Pandiaraju, S. Chen. Commun. 1998, 1639-1640. (d) Rajan, Y. C.; Kanakam, C. C. Tetwhedron Lett. 2008, 49,3023-3026. (e) Das, B.; Chowdhury, N.; Damodar, K.; Banerjee, I. Chen. Phomm. Bull. 2007, 5.5, 1274-1276.

2. For the synthesis of similar flavanone derivatives, see: (a) Skouta, R.; Li, C.-J. Angev: Chem. Int. Ed. 2007, 46, 1117-1119. (b) Nakamura, T.; Hara, O.; Tamura, T.; Makino, K.; Hamada, Y. Sinlett 2005, 155-157. (c) Hodgetts, K. J. Tetrohedron 2005, 61, 6860-6870. (d) Kawasaki, M.; Toyooka, N.; Matsui, Y.; Tanaka, A.; Goto, M.; Kakuda, H.; Kawabata, S.; Kometani, T. Heterocycles 2005, 6.5, 761-765. (e) Grigg, R; Liu, A.; Slaw, D.; Selvaratmam, S.; Woodall, D. E.; Yoganathan, G Tetrahedron Lett. 2000, 41, 7125-7128.

3. For the synthesis and synthetic usefulness of 3-aroylflavones and related compounds, see: (a) Sosnovskikh, V. Y.; Irgashev, R. A.; Kodess, . M. I. Tetrahethon 2008, 64, 2997-3004 (b) Biddle, M. M.; Lin, M.; Scheidt, K. A. $J$. Am. Chem. Soc. 2007, $129,3830-3831$. (c) Skouta, R.; Li, C.J. Tetrahedron Lett. 2007, 48, 8343-8346.

4. For the general review on Baylis-Hillman reaction, see: (a) Basavaiah, D.; Rao, A. J.; Satyanarayana, T. Chem. Rev. 2003, 103, 811-891. (b) Kim, J. N.; Lee, K. Y. Cum. Org. Chem. 2002, 6, 627-645. (c) Lee, K. Y.; Gowrisankar, S.; Kim, J. N. Bull. Korean Chem. Soc. 2005, $26,1481-1490$. (d) Singh, V.: Batra, S. Tetrahedron 2008, 64, 4511-4574 and firther references cited therein.

5. For our recent contributions on Baylis-Hillman chemistry, see: (a) Kim, S. J.; Kim, H. S.; Kim, T. H.; Kim, J. N. Bull. Korecm Chen. Soc. 2007, 28, 1605-1608. (b) Kim, H. S.; Kim, S. H.; Kim, J. N. Bull. Korean Chem. Soc. 2007, 28, 1841-1843. (c) Lee, H. S.; Kim, S. H.; Kim, T. H.; Kim, J. N. Tetrahedron Lett. 2008, 49, 1773-1776. (d) Kim, S. H.; Kim, K. H.; Kim, H. S.; Kim, J. N. Tetwhedron Lett. 2008, 49, 1948-1951.

6. (a) Kim, S. I.; Lee, H. S.; Kim, J. N. Tetrahedon Letr. 2007, 48, 1069-1072. (b) Kim, S. C.; Lee, H. S.; Kim, J. N. Bull. Konean Chem. Soc. 2007, $28,147-150$.

7. For the oxidation of 3-arylideneflavanones into 3-aroylflavones, see: (a) Nemes, C.; Levai, A.; Patonay, T.; Toth, G; Boros, S.; Halasz, J.; Adam, W; Golsch, D. J. Org. Chem. 1994, 59, 900905. (b) Mallik, A.; Chattopadhyay, F. Indion J. Chem. 1999, 38B, 889-892. (c) Mallik, A.; Chattopadhyay, F. Indicn J. Chen. 2005, 44B, 1947-1949. (d) Chawla, H. M.; Shanma S. K. Sinth. Commm. 1990, 20, 301-306. (e) Chawla, H. M.; Sharma, S. K. Bull. Soc. Chim. Fr. 1990, 127, 656-659. (f) Adam, W.; Halasz, J.; Levai, A.; Nemes, C.; Patonay, T.; Toth, G. Liebigs Am. Chen. 1994, 795803. (g) Dhande, V. P.; Thakwani, P.; Marathe, K. G. Tetrahedron $1988,44,3015-3023$.

8. For the synthesis and biological activities of 3-benzylflavone and related compounds, see: (a) Kirkiachariau, B. S.; Gomis, M. Sinth. Commmn. 2005, 35, 563-569. (b) Patonay, T.; Dinya, Z.; Levai, A,; Molnar, D. Tetrahedron 2001, 57, 2895-2907. (c) Hoshino, Y; Takeno, N. Bull. Chem. Soc. Jpn. 1994, 67, 28732875. (d) Tait, S.; Salvati, A. L.; Desideri, N.; Fiore, L. Antiviral Res. 2006, 72, 252-255. (e) Kirkiacharian, S.; Tongo, H. G.; Bastide, J.; Bastide, P.; Grenie, M. M. Eurr. J. Med. Chem. 1989 , 24, 541-546. (f) Kim, J. H.; Kim, K. H.; Kim, J. H.; Yu, Y. S.; Kim, Y.M.; Kim, K.-W.; Kwon, H. J. Biochem. Biophns. Res. Conmmin. 2007, 362, 848-852.

9. (a) Krishnamoorthy, T. V.; Rajagopalan, K.; Balasubramanian, K. K. Tetrahedron Lett. 1985, 26, 1747-1748. (b) Ashok, D.; Pallavi, K.: Reddy, G. J.; Rao, K. S. Indian J. Heterocvlic Chem. 2006, 16,95-96. (c) Ingle, T. R.; Phalnikar, N. L.; Bhide, B. V. J. Indian Chen. Soc. 1949, 26, 569-574. (d) Sangwan, N. K.; Kelkar, P. M.; Rastogi, S. N.; Anand, N. Indion J. Chem. 1985, 24B, 639-644. 